\title{
Collision Estimation for a Flexible Cantilevered-beam Subject to Random Disturbance
}

\author{
Yuichi Sawada \\ Kyoto Institiute of Technology \\ Department of Mechanical and System Engineering \\ Matsugasaki, Sakyo \\ Kyoto 606-8585, JAPAN \\ E-mail: sawada@ipc.kit.ac.jp
}

\begin{abstract}
This paper proposes a state estimation filter for detecting collisions with the flexible cantileveredbeam and obstacles. The beam is assumed to be subject to distributed random disturbance and unknown collision input. The purpose of the estimator is to detect the collision input and to estimate the strength of it. In this paper, a working model for the collision input in modal representation is considered for detection and estimation of the collision input and its strength. By applying the disturbance estimation technique, the state estimator for the collision input is constructed.
\end{abstract}

\section{INTRODUCTION}

Collisions with obstacles or moving objects, e.g., machines, desks, walls, mobile robots, human operators, and so on are serious problems in operation of flexible manipulators in working space. The collision detection and avoidance will play a significant role for such flexible manipulators. Furthermore to estimate how strong the obstacles collide with the manipulator is also important task. If a collision with an obstacle and the flexible manipulator occurs, the controller for stopping the manipulator requires a signal that indicates when the collision occurs. Then the controller must make an emergency stop so that the damage to the obstacle and the manipulator is minimal.

Approaches to detection of the collisions for the flexible manipulators are to find abrupt changes in observation data. Many researchers have done a lot of investigations for collision detections.

\footnotetext{
${ }^{1}$ Part of this research is supported by the Ministry of Education, Culture, Sports, Science and Technology of Japan under the Grants-in-Aid for Scientific Research for Encouragement of Young Scientists (B)-14750160.
}

Moorehead and Wang proposed a simple collision detection algorithm based on dynamic changes of strains of a flexible beam [1]. The detection method based on the force control of single-link robot arms has been proposed by Garcia and Feliu [2]. Kaneko, Kanayama and Tsuji investigated a kind of sensor to detect contact location [3], which is based on the static deformation of a flexible cantilevered-beam. The author proposed a collision detection method for the flexible beams based on model based fault diagnosis technique [4], [5]. On the other hand, [1] describes the measurement method for estimation of collision strength.

This paper presents an estimation filter for collision input for flexible cantilevered-beam subject to distributed random disturbance. The mathematical model of the flexible cantilevered-beam is described by the Euler-Bernoulli type partial differential equation (P.D.E.). The observation data is assumed to be measured by displacements, gradients, bending moments and/or shearing forces. In order to construct the state estimator, the mathematical model described by the P.D.E. is transformed into the modal representation model by the modal expansion method. Since the collision input is unknown and nonGaussian disturbance, its dynamics is also unknown. In this paper, a working model for the collision input in modal representation is considered for detection and estimation of the collision input and its strength. Applying the disturbance estimation method [6], [7] to the state estimator for the system model including the working model of the collision, the estimation of the collision input is achieved.

The following section presents the mathematical model of the flexible cantilevered-beam with distributed random disturbance and unknown col- 
lision input. Furthermore the mathematical model is reduced into the finite-dimensional model in modal representation. In Section III, the working model describing the behavior of the collision input is introduced. Section IV presents the augmented system consisting of the state of the flexible beam and the state of the working model of the collision input. This section is also proposed the estimation filter for the collision input based on the Kalman filter. Finally the numerical simulation results are shown in Section V.

\section{Mathematical and System Models}

In this paper, we consider to detect collisions with a flexible cantilevered-beam subject to distributed random disturbance and environmental obstacles and to estimate strength of the collision. The mathematical model of the flexible cantilevered-beam subject to the distributed random disturbance and the collision force can be described by

$$
\begin{gathered}
\rho S \frac{\partial^{2} u(t, x)}{\partial t^{2}}+c_{D} I \frac{\partial^{5} u(t, x)}{\partial x^{4} \partial t}+E I \frac{\partial^{4} u(t, x)}{\partial x^{4}} \\
=g\{\gamma(t, x)+s(t, x)\}, \quad(0 \leq x \leq \ell),
\end{gathered}
$$

where $u(t, x)$ denotes the transverse displacement from its equilibrium state of the flexible beam at time $t$ and position $x ; \gamma(t, x)$ the distributed random disturbance modeled by the white Gaussian noise; $\rho$ the mass density; $S$ the cross sectional area of the flexible beam; $E$ the Young's modulus; $I$ the second moment of cross sectional area; $c_{D}$ the coefficient of the Kelvin-Voigt damping; $g$ a constant. $s(t, x)$ represents the spatial distribution of the impact force due to the collision, which is assumed to be unknown and non-Gaussian input. The spatial distribution of $s(t, x)$ is defined by

$$
s(t, x)\left\{\begin{array}{l}
\neq 0 \text { for } t_{c} \leq t<t_{e} \\
=0 \text { otherwise }
\end{array}\right.
$$

and $t_{c}, t_{e}$ are unknown. The boundary conditions of (1) are given by

$$
u(t, 0)=\frac{\partial u(t, 0)}{\partial x}=\frac{\partial^{2} u(t, \ell)}{\partial x^{2}}=\frac{\partial^{3} u(t, \ell)}{\partial x^{3}}=0 .
$$

The $j$-th observation is described by

$$
\begin{gathered}
y_{j}(t)=a_{j} u\left(t, \xi_{j}\right)+b_{j} \frac{\partial u\left(t, \xi_{j}\right)}{\partial t} \\
+c_{j} \frac{\partial^{2} u\left(t, \xi_{j}\right)}{\partial x^{2}}+d_{j} \frac{\partial^{3} u\left(t, \xi_{j}\right)}{\partial x^{2} \partial t}+e_{j} \beta_{j}(t), \\
j=1, \cdots, P
\end{gathered}
$$

where $0 \leq \xi_{1}<\xi_{2}<\cdots<\xi_{P} \leq \ell ; a_{j}, b_{j}, c_{j}, d_{j}$ and $e_{j}$ are non-negative constant; $\beta_{j}(t)$ the observation noise modeled by the white Gaussian noise.

The solution of (1) is described by

$$
u(t, x) \cong \sum_{k=1}^{N} u_{k}(t) \phi_{k}(x)
$$

where $N$ is assumed to be sufficiently large positive number; $\phi_{k}(x)$ are mode functions corresponding to the eigenvalues $\lambda_{k}$ with respect to the eigenvalue problem $\mathcal{A} \phi_{k}(x)=\lambda_{k} \phi_{k}(x), \mathcal{A}:=$ $(E I / \rho S) d^{4} / d x^{4} . \phi_{k}(x)$ are the orthonormal functions, i.e., $\left\langle\phi_{k}, \phi_{j}\right\rangle=\delta_{k j}$, where $\delta_{k j}$ denotes the Kronecker delta; and the inner product is defined by $\langle f, g\rangle:=\int_{0}^{\ell} f(x) g(x) d x$. Substituting (5) into (1) and taking the inner product with $\phi_{j}(x)$ both side, we have a modal representation models described by

$$
\begin{aligned}
\ddot{u}_{k}(t)+\frac{c_{D}}{E} \lambda_{k} \dot{u}_{k}(t)+\lambda_{k} u_{k}(t) & \\
= & \frac{g}{\rho S} \gamma_{k}(t)+\frac{g}{\rho S} s_{k}(t), \\
k & =1, \cdots, N
\end{aligned}
$$

where

$$
\begin{gathered}
\gamma_{k}(t)=<\gamma(t, \cdot), \phi_{k}> \\
s_{k}(t)=<s(t, \cdot), \phi_{k}>.
\end{gathered}
$$

Introducing a state vector $v_{N}(t):=\left[\tilde{u}_{N}^{\prime}(t)\right.$, $\left.\dot{\tilde{u}}_{N}^{\prime}(t)\right]^{\prime}$, where $\tilde{u}_{N}(t):=\left[u_{1}(t), u_{2}(t), \cdots, u_{N}(t)\right]^{\prime}$, the modal representation models described by $(6)$ is written into the state space model:

$$
\dot{v}_{N}(t)=A_{N} v_{N}(t)+G_{N}\left\{\gamma_{N}(t)+\sigma_{N}(t)\right\},
$$

where $\gamma_{N}(t):=\left[\gamma_{1}(t), \gamma_{2}(t), \cdots, \gamma_{N}(t)\right]^{\prime} ; \sigma_{N}(t):=$ $\left[s_{1}(t), s_{2}(t), \cdots, s_{N}(t)\right]^{\prime}$, and

$$
A_{N}=\left[\begin{array}{cc}
0 & I_{N} \\
-\Lambda & -\frac{c_{D}}{E} \Lambda
\end{array}\right], \quad G_{N}=\left[\begin{array}{c}
0 \\
\frac{g}{\rho S} I_{N}
\end{array}\right],
$$

and where $\Lambda=\operatorname{diag}\left\{\lambda_{1}, \cdots, \lambda_{N}\right\}$ and $I_{*}$ denotes the unit matrix.

Similarly, the observation system (4) can be expressed by

$$
y(t)=C_{N} v_{N}(t)+E_{N} \beta_{N}(t),
$$




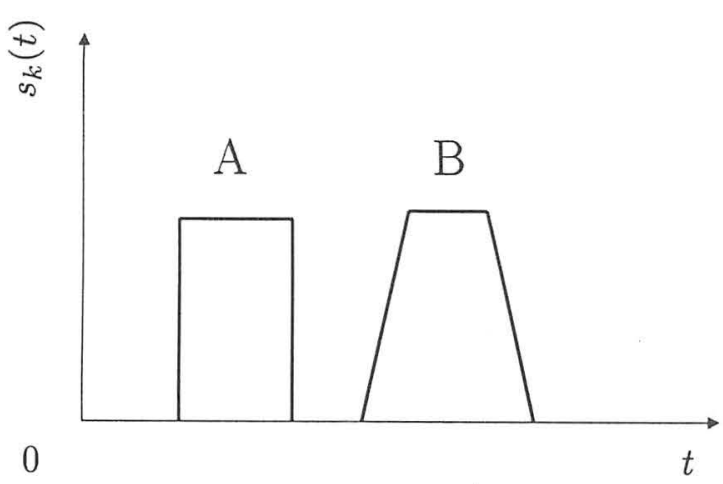

Fig. 1. Considerations of the time evolution of the collision input, $s_{k}(t), k=1, \cdots, N$.

where $y(t)=\left[y_{1}(t), \cdots, y_{P}(t)\right]^{\prime} ; \beta_{N}(t)=$ $\left[\beta_{1}(t), \cdots, \beta_{P}(t)\right]^{\prime} ; \quad C_{N}=\left[\tilde{C}_{c}, \tilde{C}_{e}\right] ; \quad E_{N}=$ $\operatorname{diag}\left\{e_{1}, \cdots, e_{P}\right\}$;

$$
\begin{aligned}
& {\left[\tilde{C}_{c}\right]_{i j}=a_{i} \phi_{j}\left(\xi_{i}\right)+c_{i} \frac{d^{2} \phi_{j}\left(\xi_{i}\right)}{d x^{2}}} \\
& {\left[\tilde{C}_{e}\right]_{i j}=b_{i} \phi_{j}\left(\xi_{i}\right)+d_{i} \frac{d^{2} \phi_{j}\left(\xi_{i}\right)}{d x^{2}} .}
\end{aligned}
$$

We rewrite the system equations (9) and (11) into the form of the Itô's stochastic differential equations, i.e.,

$$
d v_{N}(t)=A_{N} v_{N}(t) d t+G_{N}\left\{d w_{N}(t)+\sigma_{N}(t) d t\right\}
$$

$$
d y(t)=C_{N} v_{N}(t) d t+E_{N} d w_{\beta}(t)
$$

where $w_{N}(t)=\int_{0}^{t} \gamma_{N}(\tau) d \tau$ and $w_{\beta}(t)=\int_{0}^{t} \beta_{N}(\tau)$ $d \tau ; \mathcal{E}\left\{d w_{N}(t) d w_{N}^{\prime}(t)\right\}=W_{N} d t$ and $\mathcal{E}\left\{d w_{\beta}(t)\right.$ $\left.d w_{\beta}^{\prime}(t)\right\}=V_{N} d t ; \mathcal{E}\left\{d w_{N}(t) d w_{\beta}^{\prime}(t)\right\}=0$.

\section{Working Model for Collision InPUT}

The collision input $s(t, x)$ is nonGaussian and unknown input with unknown dynamics. However if we need to know when the collision occurs and how strong impact the flexible beam receives, the collision input $s(t, x)$ should be estimated. In this paper, to estimate strength of the collision input, dynamics of $s_{k}(t)$ instead of dynamics of $s(t, x)$ are considered. Here, two types of collision's motions illustrated in Fig.1 are considered. The type A is assumed to be $\dot{s}_{k}(t) \cong 0$ which is the simplest case of collision. The type B is assumed to hold $\ddot{s}_{k}(t) \cong 0$.

For both cases, $\dot{s}_{k}(t)$ and $\ddot{s}_{k}(t)$ might have small fluctuation. Hence, the system models for such collision input are considered as follows:

$$
\begin{array}{ll}
\text { Type A: } & \dot{s}_{k}=\alpha \gamma_{\alpha}(t) \\
\text { Type B: } & \ddot{s}_{k}=\alpha \gamma_{\alpha}(t),
\end{array}
$$

where $\alpha$ denotes small constant; $\gamma_{\alpha}(t)$ the white Gaussian noise. From a practical point of view, we employ (15) (type B) for the working model of the collision input.

Introducing $\sigma_{N}(t),(15)$ is described by

$$
\left[\begin{array}{c}
\dot{\sigma}_{N}(t) \\
\ddot{\sigma}_{N}(t)
\end{array}\right]=\left[\begin{array}{cc}
0 & I_{N} \\
0 & 0
\end{array}\right]\left[\begin{array}{c}
\sigma_{N}(t) \\
\dot{\sigma}_{N}(t)
\end{array}\right]+\left[\begin{array}{c}
0 \\
\alpha I_{N}
\end{array}\right] \gamma_{\alpha}(t)
$$

where $\gamma_{\alpha}(t) \in \mathbb{R}^{N}$ denotes the white Gaussian noise.

\section{Augmented System and Collision Estimation Filter}

To estimate strength of the collision input, we introduce a new state vector $v_{c}(t)$ := $\left[v_{N}^{\prime}(t), \quad \sigma_{N}^{\prime}(t), \quad \dot{\sigma}_{N}^{\prime}(t)\right]^{\prime}$. The system model with the working model given by (16) can be described by

$$
\begin{aligned}
d v_{c}(t) & =A_{c} v_{c}(t) d t+G_{c} d w_{c}(t) \\
d y(t) & =C_{c} v_{c}(t) d t+E_{N} d w_{\beta}(t),
\end{aligned}
$$

where $d w_{c}(t)=\left[d w_{N}^{\prime}(t), 0, d w_{\alpha}^{\prime}(t)\right]^{\prime} ; \quad w_{\alpha}(t)=$ $\int_{0}^{t} \gamma_{\alpha}(\tau) d \tau ; \quad \mathcal{E}\left\{d w_{\alpha}(t) d w_{\alpha}^{\prime}(t)\right\}=W_{\alpha} d t ;$ $\mathcal{E}\left\{d w_{\alpha}(t) d w_{\beta}^{\prime}(t)\right\}=0 ; \quad \mathcal{E}\left\{d w_{c}(t) d w_{c}^{\prime}(t)\right\}=$ Block diag $\left\{W_{N}, 0, W_{\alpha}\right\}=W_{c}$

$$
\begin{aligned}
A_{c} & =\left[\begin{array}{ccc}
A_{N} & G_{N} & 0 \\
0 & 0 & I_{N} \\
0 & 0 & 0
\end{array}\right], G_{c}=\left[\begin{array}{cc}
G_{N} & 0 \\
0 & 0 \\
0 & \alpha I_{N}
\end{array}\right] \\
C_{c} & =\left[\begin{array}{lll}
C_{N} & 0 & 0
\end{array}\right] .
\end{aligned}
$$

The collision estimation filter is constructed by a Kalman filter based on the augmented system (17) and (18). The Kalman filter is given by

$$
\begin{gathered}
d \hat{v}_{c}(t)=A_{c} \hat{v}_{c}(t) d t+K\left\{d y(t)-C_{c} \hat{v}_{c}(t) d t\right\} \\
K=P C_{c}^{\prime}\left(E_{c} V_{N} E_{c}^{\prime}\right)^{-1} \\
A_{c} P+P A_{c}^{\prime}-P C_{c}^{\prime}\left(E_{c} V_{N} E_{c}^{\prime}\right)^{-1} C_{c} P \\
\quad+G_{c} W_{c} G_{c}^{\prime}=0
\end{gathered}
$$

where $\hat{v}_{c}(t):=\mathcal{E}\left\{v_{c}(t) \mid \mathcal{Y}_{t}\right\}, \mathcal{Y}_{t}=\{y(s) \mid 0 \leq s \leq t\}$ denotes the state estimate; $\mathcal{E}\left\{\cdot \mid \mathcal{Y}_{t}\right\}$ the conditional expectation. 
Table 1. The parameters for the collisions.

\begin{tabular}{|c|c|c|c|c|c|}
\hline Cases & $t_{c}(\mathrm{~s})$ & $t_{e}(\mathrm{~s})$ & $f_{r}(\mathrm{~N} / \mathrm{m})$ & $x_{c}(\mathrm{~m})$ & $x_{w}(\mathrm{~m})$ \\
\hline \hline Case 1 & 5 & 5.2 & 0.50 & 0.56 & 0.02 \\
Case 2 & 5 & 5.8 & 0.50 & 0.56 & 0.02 \\
Case 3 & 5 & 5.5 & 0.15 & 0.90 & 0.02 \\
\hline
\end{tabular}

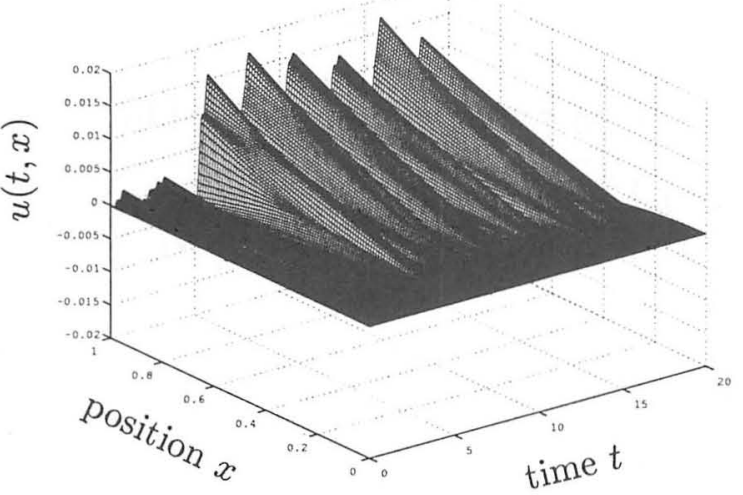

Fig. 2. The vibration of flexible cantilevered-beam (Case 1).

\section{Simulation Results}

In this paper, several numerical simulations have been performed. The flexible cantilevered-beam is assumed to have the length $\ell=1(\mathrm{~m})$, the Young's modulus $E=1.96 \times 10^{5}(\mathrm{MPa})$, the second moment of cross sectional area $I=4.28 \times 10^{-13}(\mathrm{~m})^{4}$, the cross sectional area $S=2.03 \times 10^{-5}\left(\mathrm{~m}^{2}\right)=$ $4.04 \times 10^{-2} \times 5.03 \times 10^{-4}\left(\mathrm{~m}^{2}\right)$, the mass density $\rho=7.86 \times 10^{3}\left(\mathrm{~kg} \cdot \mathrm{m}^{-3}\right)$, the Kelvin-Voigt damping $c_{D}=4.82 \times 10^{8}\left(\mathrm{~N} \cdot \mathrm{s} \cdot \mathrm{m}^{-2}\right)$. Three displacement sensors are installed at $\xi_{1}=0.5(\mathrm{~m}), \xi_{2}=0.75(\mathrm{~m})$, $\xi_{3}=1(\mathrm{~m})$. The other parameters were set as $c_{j}=20, e_{j}=1 \times 10^{-4},(j=1, \cdots, 3), g=0.125$ and $\alpha=0.5$. As the numerical model of the flexible cantilevered-beam, the first eight modes, $u_{k}(t)(k=1, \cdots, 8)$, are used. The Kalman filter was designed for the first three modes, i.e., $u_{k}(t)(k=1, \cdots, 3)$, i.e., $N=3$. The time division in the numerical simulation was set as $\Delta t=0.0278(\mathrm{~s})$.

The collision input was considered as follows:

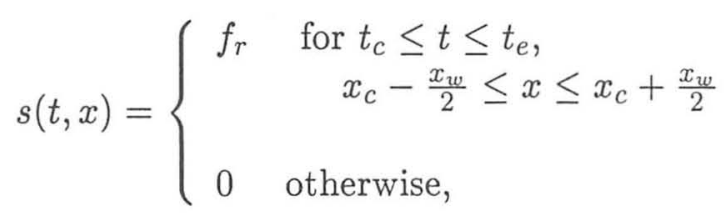

where $f_{r}, x_{c}$ and $x_{w}$ are constant.

In this section, three cases of collision inputs are used. The parameters for the collisions, $f_{r}, x_{c}$ and $x_{w}$, are listed in Table 1 .

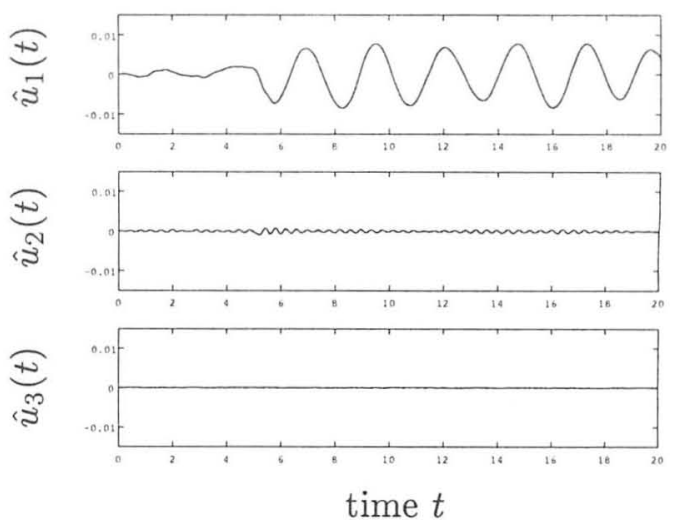

Fig. 3. The state estimate $\hat{u}_{c}(t)$ (Case 1$)$.

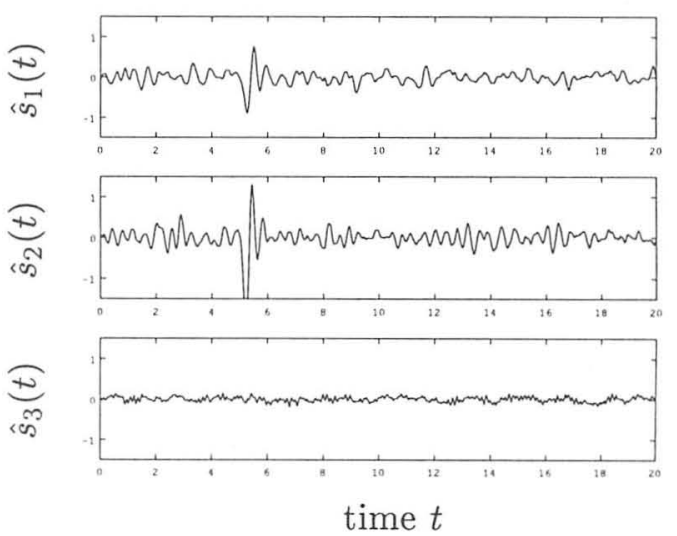

Fig. 4. The state estimate of the collision input $\hat{\sigma}_{N}(t)$ (Case 1).

Case 1: This case is assumed that the obstacle collides at $x=0.56 \mathrm{~m}$ (see Table 1). Figs. 2 and 3 show the vibration of the flexible cantileveredbeam subject to distributed random disturbance and the state estimate of the flexible beam, respectively. It can be seen that the collision occurred at $t=5(\mathrm{~s})$ makes a large amplitude vibration. The estimation of the collision input in modal representation, $s_{k}(t)$, is depicted in Fig.4. There appear large amplitudes at around $t=5(\mathrm{~s})$ in the figures of $\hat{s}_{1}(t)$ and $\hat{s}_{2}(t)$. The estimate of spatially distributed collision input $\hat{s}(t, x):=\sum_{k=1}^{N} \hat{s}_{k}(t) \phi_{k}(x)$ is shown in Fig.5, which reveals that the flexible beam receives large force due to the collision at around the center of the beam.

Case 2: This case is that the obstacle collides with the flexible beam for $0.8(\mathrm{~s})$. (The collision in Case 1 is for $0.2(\mathrm{~s})$.) The vibration of the flexible beam and the state estimate are shown in Figs. 6 and 7. As seen in Fig.8, the proposed estimator indicates that the collision occurs from $t=5(\mathrm{~s})$ to $6(\mathrm{~s})$. Fig.9 also shows that the collision input affects at around the center of the beam. 


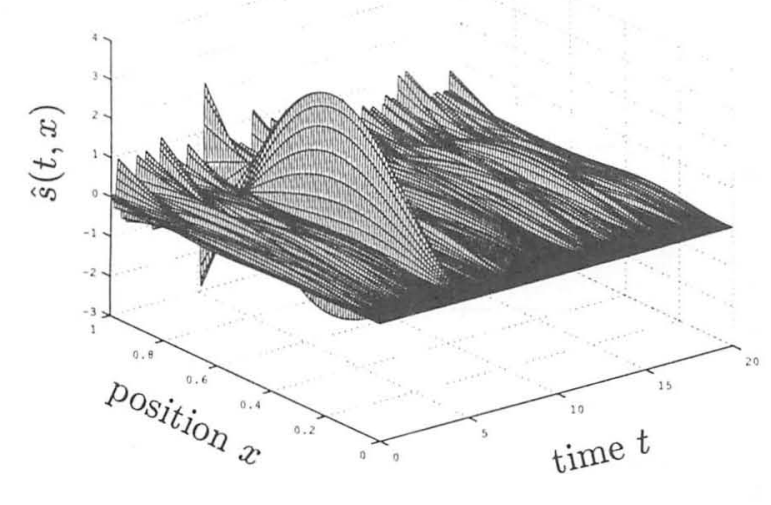

Fig. 5. The time evolution of estimate of the collision input $\hat{s}(t, x)=\sum_{k=1}^{N} \hat{s}_{k}(t) \phi_{k}(x)$ (Case 1 ).

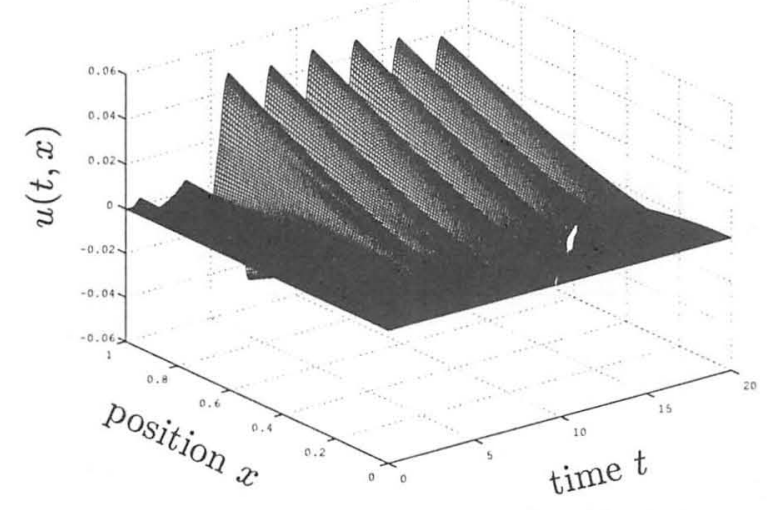

Fig. 6. The vibration of flexible cantilevered-beam (Case 2).

Case 3: In this Case, a weak collision occurs at $x_{c}=0.9 \mathrm{~m}$. The vibration of the beam is smaller than in Case 1 and 2 (see Figs. 10 and 11). Fig. 12 depicts the estimate of the collision input $\hat{s}_{k}(t)$. There appear signals of the collision at $t=5(\mathrm{~s})$ in the noisy processes. However, we can see large amplitude of $\hat{s}(t, x)$ near the tip of the flexible beam illustrated in Fig.13.

\section{CONCLUSIONS}

The Kalman filter based estimator for detecting the collision with unlooked-for obstacle and the flexible cantilevered-beam subject to distributed random disturbance has been proposed. By assuming the behavior of the collision input and introducing the working model for the collision input, the estimation of the spatial distribution of the collision input has been achieved. The proposed method indicates when the collision occurs and how strong the obstacle collides with the flexi-

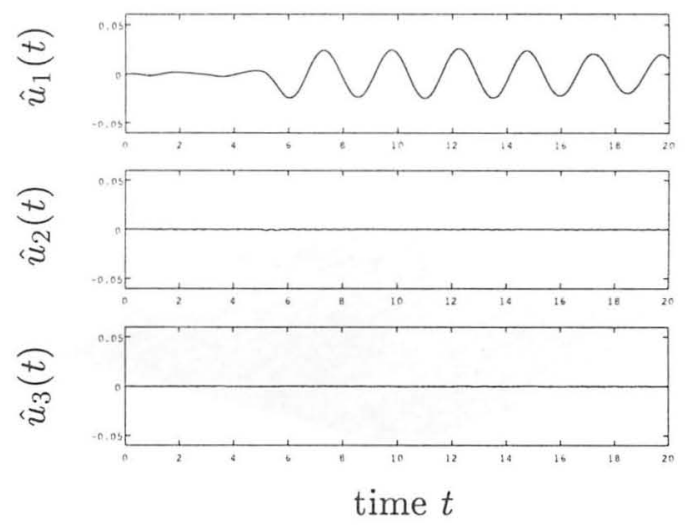

Fig. 7. The state estimate $\hat{u}_{c}(t)$ (Case 2).

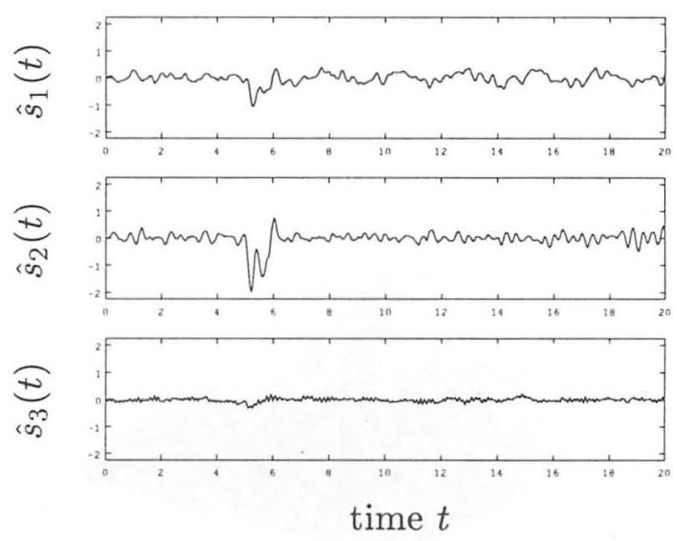

Fig. 8. The state estimate of the collision input $\hat{\sigma}_{N}(t)$ (Case 2).

ble beam in the sense of modal representation. The simulation studies have shown that the estimate of collision input $\hat{s}(t, x)$ has clearly represented which part of the beam has strongly been received the force due to the collision.

\section{REFERENCES}

[1] S. Moorehead and D. Wang: Collision Detection Using a Flexible Manipulator: A Feasibility Study, Proc. of the 1996 IEEE Internatioal Conference on Robotics and Automation, 804/809 (1996)

[2] A. Garcia and V. Feliu: Force Control of a Flexible Robot Based on a Collision Detection Mechanism, IEE Proc. of the Control Theory and Applications, 147, No.6, 588/595 (2000)

[3] M. Kaneko, N. Kanayama and T. Tsuji: Active Antenna for Contact Sensing, IEEE Trans. on Robotics and Automation, 14, No.2, 278/291 (1998)

[4] Y. Sawada: Detection of Collisions for a Flexible Beam Subject to Random Disturbance, Proc. of SICE Annual Conference 2002 in Osaka, 268/273 (2002)

[5] Y. Sawada: Collision Detection for a Flexible Cantilever-beam Subject to Random Disturbance Based on Innovation Process, Proc. of IEEE Control Systems Society Conference on Control Applications, Glasgow, 1171/1176 (2002) 


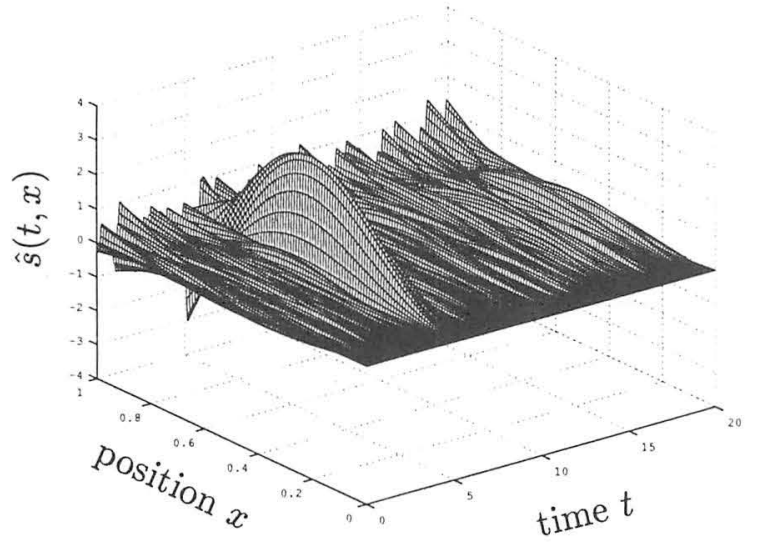

Fig. 9. The time evolution of estimate of the collision input $\hat{s}(t, x)=\sum_{k=1}^{N} \hat{s}_{k}(t) \phi_{k}(x)$ (Case 2$)$.

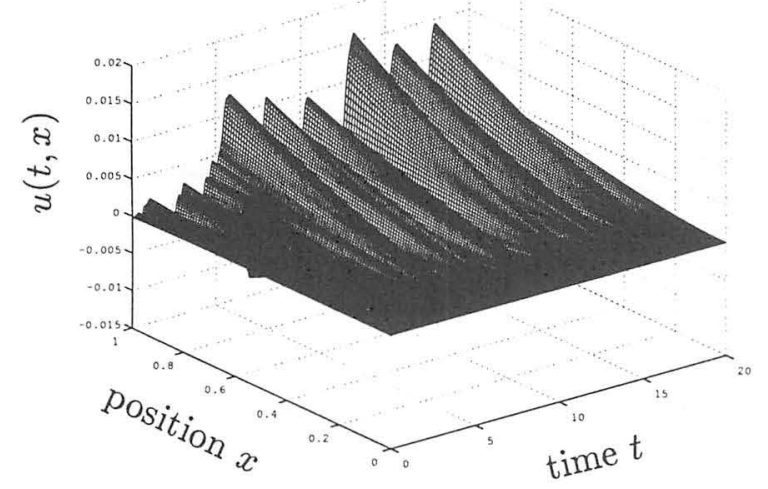

Fig. 10. The vibration of flexible cantilevered-beam (Case 3).

[6] C.-F. Lin, A. G. Ulsoy and D. J. LeBlanc: Vehicle Dynamics and External Disturbance Estimation for Vehicle Path Prediction, IEEE Trans. on Control Systems Technology, 8, No.3, 508/518 (2000)

[7] K.-H. Kim, I.-C. Baik, G.-W. Moon and M.-J. Youn: A Current Control for a Permanent Magnet Synchronous Motor with a Simple Disturbance Estimation Scheme, IEEE Trans. on Control Systems Technology, 7, No.5, 630/633 (1999)

[8] M. Massoumnia, G. C. Verghese and A. S. Willsky: Failure Detection and Identification, IEEE Trans. on Automatic Control, 34, No.3, 316/321 (1989)

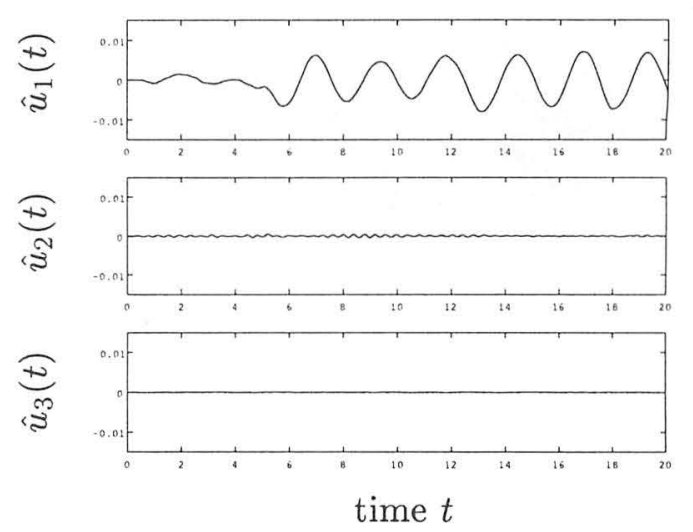

Fig. 11. The state estimate $\hat{u}_{c}(t)$ (Case 3 ).

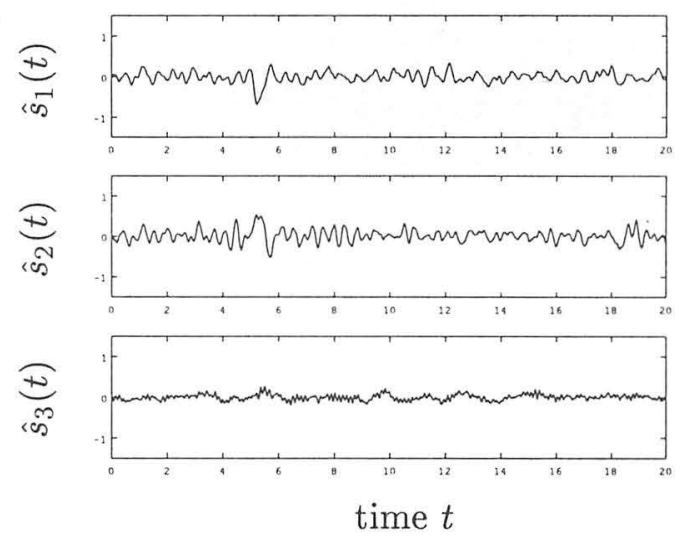

Fig. 12. The state estimate of the collision input $\hat{\sigma}_{N}(t)$ (Case 3).

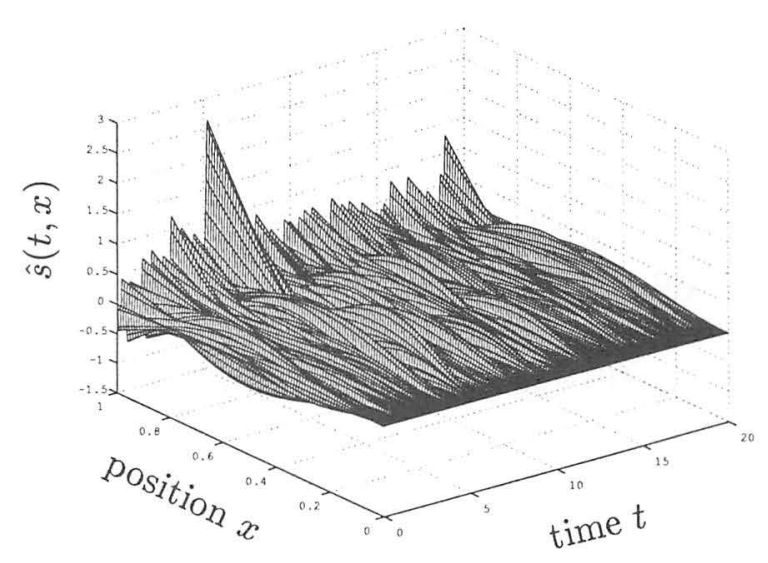

Fig. 13. The time evolution of estimate of the collision input $\hat{s}(t, x)=\sum_{k=1}^{N} \hat{s}_{k}(t) \phi_{k}(x)$ (Case 3$)$. 